\section{NEW ELEMENTS}

\section{Manipulating Nuclei at the Nucleon Level}

Systematic results accompanying the recent discovery of isotopes of elements 110 and 111 at GSI's heavy-ion accelerator combined with theoretical work indicates that it may be possible to reach element 116 using the latest techniques based on a "frictionless" fusion process at low beam energies.
The production and identification of new heavy elements has been studied extensively since heavy-ion accelerators became widely available. The most successful method is based on fusion-evaporation reactions between projectile particles and heavy metal targets, recoil separation and the identification of nuclei by delayed coincidences to known daughter decays after implantation into position-sensitive detectors [1]. One popular approach uses targets comprising thin metal layers evaporated onto carbon foils mounted on a wheel whose rotation speed is synchronised to the heavy-ion beam pulses from the accelerator. In contrast to recoil stopping, recoil separation makes use of the ionic charge and momentum of the recoiling fusion products obtained in the reaction process. Spatial separation of heavy nuclei from the projectiles and other reaction products is achieved by electric and magnetic fields. Gas-filled recoil separators use the different magnetic rigidities of recoils and projectiles traversing a lowpressure gas-filled volume. Wien filter velocity separators are based on the different rigidities of fusion products, projectiles and other reaction products after escape from solid targets into a vacuum.

Once nuclei produced in various evaporation channels have been filtered out while retaining a high transmission efficiency, the transmitted particles are identified by a detector system. Theoretical and experimental results for the stability of superheavy nuclei indicate that they decay via $\alpha$-particle emission or by fission with half-lives ranging from microseconds to days so silicon sesuitable.

If the total rate of separated ions is low, their positions can be determined by implanting the particles into position-sensitive silicon detectors. This is also the case if the implanted nuclei are radioactive because recoil effects are small compared with the ranges of implanted nuclei, emitted $\alpha$ particles and fission products. Decay chains are then recorded by analysing delayed

This report is taken from the presentation "Production and Stability of New Elements" made at the 15th EPS Nuclear Physics Divison Conference on Low Energy Nuclear Dynamics (St. Petersburg, 18-22 April 1995) by $\mathrm{S}$. Hofmann who leads the SHIP and Heavy Elements Group atf the Gesellschaft für Schwerionenforschung (GSI) $\mathrm{mbH}$, Postfach 1105 52, D-64220 Darmstadt. miconductor detectors are among the most coincidences on an event-by-event basis, using variable position and time windows, and the implanted reaction products are correlated with the radioactive decay.

The Wien-type Separator for Heavy-Ion Reaction Products (SHIP) facility (Fig. 1) at the Gesellschaft für Schwerionenforschung (GSI), Darmstadt, epitomises this extremely successful approach. Experiments resulted in the discovery late last year of two isotopes of element 110 [2] and one isotope of element 111 [3] following the discovery in 1981-84 [4] of elements 107, 108 and 109, thus bringing the number of man-made elements to 21. The work was the direct outcome of an upgrade of the SHIP facility to a sensitivity level that would be sufficient to identify heavy elements produced in reactions with cross-sections of $1 \mathrm{pb}$ and less.

Before the upgrade the estimated counting rate for a $1 \mathrm{pb}$ cross-section was an impractical 1 event per 54 days, as compared with a theoretical maximum of 0.9 events per day assuming reasonable accelerator and target properties with $100 \%$ efficient separation and detection. Achieving a rate closer to the maximum theoretical value involved installing a new high-charge injector, increasing the separator solid angle to increase the transmission of fusion reaction products, and decreasing the detector system's background in order to be able to accept higher beam currents and to increase the significance of detected correlated events.

\section{Preliminary Work}

The stability of heavy and superheavy nuclei had been studied theoretically prior

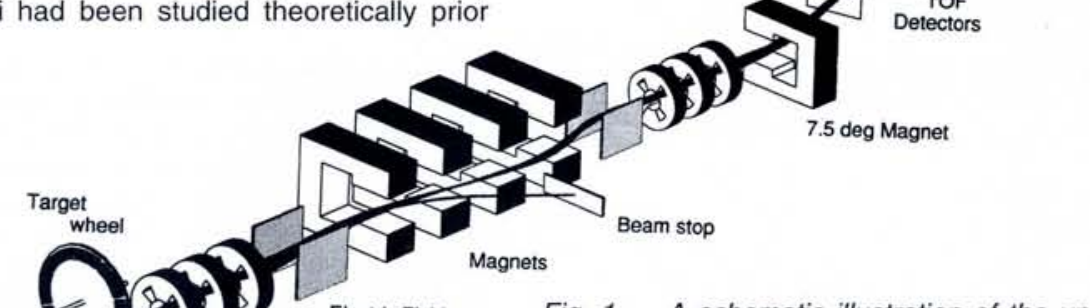

Fig. 1-A schematic illustration of the upgraded SHIP recoil separator and detection system. Pulses of projectile ions from a linear accelerator strike thin heavy-metal targets

Projectiles Lenses

Electric Field

to carrying out the experiments. Calculations of the ground-state microscopic shell correction energy revealed two minima (one of about $-8 \mathrm{MeV}$ for strongly deformed nuclei centred around 272110 ; the other of about $-9 \mathrm{MeV}$ around 292114 for an island of spherical superheavy nuclei). Known data was well reproduced and it was concluded that most nuclei which can be produced with stable projectiles and available targets should be $\alpha$-particle emitters with half-lives larger than $1 \mu$ s and therefore easily accessible in experiments using recoil separators.

However, it was unclear if the $1 \mathrm{pb}$ crosssection limit would be sufficient for detecting elements above 109 using systems like ${ }^{48} \mathrm{Ca}+{ }^{248} \mathrm{Cm}$ system which had been employed in earlier experiments. The GSI team therefore decided to adopt a safer strategy by using heavier projectiles and targets. However, the excitation functions for the production of heavy elements at projectile energies close to the Coulomb barrier are very narrow, as a function of the excitation energy, so the optimum projectile energies had first to be determined. Reaction theories predict that so-called "extrapush" energy is needed to fuse heavy particles. It results in a higher excitation energy of the compound nucleus, leading to a decrease in fusion cross-section owing to prompt fission.

Production cross-section maxima are determined by the competition between mounted on a rotating wheel. The evaporation residues which are produced are separated in flight by an electromagnetic velocity filter and a dipole magnet deflects the separated products into a detector system comprising two time-of-flight (TOF) detectors and a box-like arrangement of silicon detectors followed by germanium detectors. The TOF detectors distinguish implantations from radioactive decays of previously implanted nuclei, the silicon detectors are position sensitive, and the germanium detectors measure $X$-rays or $\gamma$-rays in coincidence with the silicon detectors. 
Fig. 2 - Experimental maximum cross-sections of residues after fusion reactions and evaporation of one neutron for targets bombarded with projectiles of equal or similar isospin (the lines are least square fits and extrapolations).

complex fusion probabilities and prompt fission [5] so the optimum beam energies could not be estimated accurately for superheavy elements. Moreover, extrapush energies for asymmetric reactions with elements down to element 103 were unknown so it was impossible to make extrapolations. Excitation functions for fusion-evaporation reactions with ${ }^{208} \mathrm{~Pb}$ and ${ }^{209} \mathrm{Bi}$ targets and ${ }^{50} \mathrm{Ti}$ and ${ }^{58} \mathrm{Fe}$ projectiles were therefore measured. The optimum beam energy for the production of element 110 by the reaction $62 \mathrm{Ni}+{ }^{208} \mathrm{~Pb} \rightarrow{ }^{269} 110+1$ neutron was then obtained by linear extrapolation.

\section{Experiments}

After experiments at the optimum beam energy to produce element 110 , the projectile ions were changed to ${ }^{64} \mathrm{Ni}$ and the target to ${ }^{208} \mathrm{~Pb}$ and ${ }^{209} \mathrm{Bi}$ in aiming to produce element 110 using five different beam energies and element 111 using two different beam energies, respectively. Rare decay processes involving several generations of $\alpha$-decays were revealed by delayed $\alpha-\alpha$ coincidence spectra obtained by accumulating $\alpha$-decays for which: a) all signals originated from the same position in the detector; b) a high-energy (approximately $25 \mathrm{MeV}$ ) implantation signal occurred within a time window of typically $20 \mathrm{~ms}$ before implantation and in coincidence with the signals from SHIP's time-of-flight detectors; c) at least one member of the decay chain followed within subsequent time windows of typically $2 \mathrm{~s}, 20 \mathrm{~s}$ and $200 \mathrm{~s}$ duration. In particular, the initial experiments with the ${ }^{62} \mathrm{Ni}+{ }^{208} \mathrm{~Pb}$ system revealed a decay chain
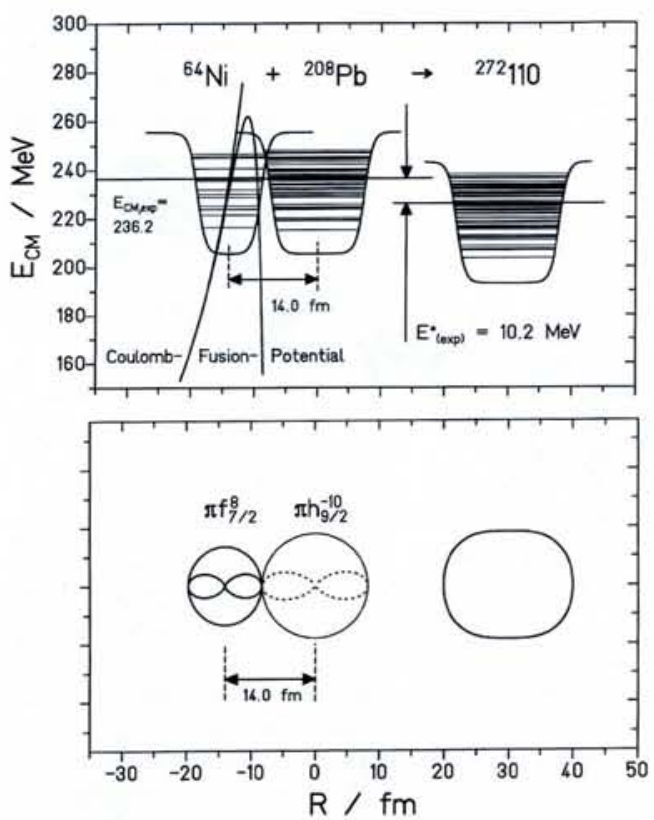

Fig. 3 - Fusion initiated by transfer. The reaction between a spherical ${ }^{208} \mathrm{~Pb}$ target nucleus and a ${ }^{64} \mathrm{Ni}$ projectile product at the experimental centre-of-mass energy $E_{C M \text {,exp }}$ of 236.2 MeV which resulted in the deformed 272110 fusion product. The upper panel gives an energy-distance diagram for nuclei brought to rest at the (measured) maximum crosssection. The occupied levels at the Fermi surface are just in contact so nucleons or pairs of nucleons leave the orbit of one nucleus and move into the free orbit of the reaction partner (illustrated schematically in the lower panel showing proton orbitals at the contact point). The Coulombic repulsion force is reduced so a compound nucleus with an excitation energy $E_{(\exp )}^{*}$ of only $10.2 \mathrm{Mev}$ forms. partners become so strong that even at moderate angular momenta a compound system cannot be formed and the reaction partners simply reseparate. However, the largest measured cross-sections for the two new elements were observed at energies well below the classical fusion barrier so a tunnelling process cannot account for the cross-sections. Meanwhile, a semiclassical approach gives a tunnelling probability that is too small to contribute to the measured cross-section.

The measured reaction properties can be explained in terms of fusion initiated by a low-energy rearrangement process involving the transfer of nucleons. Consider reactions comprising the head-on collision of spherical target nuclei and nearly spherical projectiles that proceed at extremely low dissipative energies. One can show that when the reaction partners come to rest owing to Coulomb repulsion at the separation distance corresponding to the maximum in the production cross-section, the occupied energy levels at the surface of the nucleus are on the point of contacting. The kinetic energy of orbiting nucleons is low at the Fermi surface so at the moment of contact, the probability is the highest for nucleons or pairs of nucleons to leave the orbit of one nucleus and move into a free orbit of the reaction partner. This frictionless transfer process is especially favourable in the case of a $\mathrm{Pb}$ target. In $208 \mathrm{~Pb}$, the $h_{11 / 2}$ proton subshell is filled and the free orbits are for $h_{9 / 2}$ protons. Proton transfer profits from the proton-proton attraction on filling the $h_{9 / 2}$ subshell. Similarly, neutron transfer profits from neutron-neutron attraction in volving other orbits and subshells.

The transfer of protons, which resembles a one-step resonant capture process, essentially reduces the Coulomb barrier thus allowing the reaction partners to remain in close contact and to fuse. The emission of a neutron cools the system down to a level of excitation which allows the final nucleus to profit fully from shell stabilisation. Using the model one estimates that in the ${ }^{82} \mathrm{Se}+$ ${ }^{208} \mathrm{~Pb}$ system, the superheavy nucleus 290116 will probably be produced in its ground state or at a very low excitation energy as dissipative energies approach zero with increasing atomic number.

Provided the half-life of 290116 remains within the $10 \mu \mathrm{s}-100 \mathrm{~ms}$ range, we shall know more when the SHIP facility starts operating again towards the end of 1995. More generally, the conclusion is that the field of superheavy element production now encompasses low-energy nuclear dynamics phenomena ranging from well-ordered $\mathrm{mi}$ croscopically determined systems to processes governed by statistical disorder.

[1] Hofmann S., J. Alloys \& Compounds 213/214 (1994) 74.

[2] Hofmann S. et al., Z. Physik A 350 (1995) 277.

[3] ibid, 350 (1995) 281

[4] Münzenberg G. et al., Z. Phys. A 300 (1981) 107, ibid 317 (1984) 235, ibid, 309 (1982) 89.

[5] Aguiar C.E. et al., Nucl. Phys. A 491 (1989) 301; Aguiar C.E. idem., 517 (1990) 205.

[6] Hofmann S., GSI Preprint GSI-95-25 (1995). 